\title{
Average-Case Competitive Analyses for One-Way Trading
}

\author{
Hiroshi Fujiwara ${ }^{1}$ \\ Toyohashi University of Technology \\ Kazuo Iwama \\ Yoshiyuki Sekiguchi \\ Kyoto University Tokyo University of Marine Science and Technology
}

\begin{abstract}
Consider a trader who exchanges one dollar into yen and assume that the exchange rate fluctuates within the interval $[m, M]$. The game ends without advance notice, then the trader is forced to exchange all the remaining dollars at the minimum rate $m$. El-Yaniv et al. presented the optimal worst-case threat-based strategy for this game [EFKT01]. In this paper, under the assumption that the distribution of the maximum exchange rate is known, we provide average-case analyses using all the reasonable optimization measures and derive different optimal strategies for each of them. Remarkable differences in behavior are as follows: Unlike other strategies, the average-case threat-based strategy that minimizes $E[\mathrm{OPT} / \mathrm{ALG}]$ exchanges little by little. The maximization of $E[\mathrm{ALG} / \mathrm{OPT}]$ and the minimization of $E[\mathrm{OPT}] / E[\mathrm{ALG}]$ lead to similar strategies in that both exchange all at once. However, their timing is different. We also prove minimax theorems with respect to each objective function.
\end{abstract}

Keywords. Online Algorithms, Competitive Analysis, Average-Case Analysis, Stochastic Analysis, Functional Analysis, Currency Trading, One-Way Trading, Financial Engineering.

\section{Introduction}

Since online competitive analysis was introduced, there has scarcely been any significant difference in attitude to worst-case performance evaluation of online algorithms. Most studies use, as their performance measure, $\max \left[\frac{\mathrm{OPT}}{\mathrm{ALG}}\right]$ or $\min \left[\frac{\mathrm{ALG}}{\mathrm{OPT}}\right]$ for maximization problems, where ALG and OPT denote the returns for maximization (the costs for minimization) of an online and an optimal offline algorithms, respectively. (The difference between the two is not essential, both are getting better with the quantity approaching to 1.0.) When it comes to average-case evaluation with an input distribution, what is an adequate measure? A natural extension based on expectations would be $E\left[\frac{\mathrm{OPT}}{\mathrm{ALG}}\right], \frac{E[\mathrm{OPT}]}{E[\mathrm{ALG}]}, E\left[\frac{\mathrm{ALG}}{\mathrm{OPT}}\right]$, or $\frac{E[\mathrm{ALG}]}{E[\mathrm{OPT}]}$. It might seem that all of them are reasonable and which one to be used is just a matter of taste. In this paper we warn that such an easy choice is in fact quite dangerous by illustrating that the resulting optimal algorithm varies crucially depending on which measure is adopted.

Our problem in this paper is one-way trading [EFKT01]. In this problem a trader owns one dollar at the beginning and exchanges it into as much yen as possible, depending on the exchange rate. We assume the range of the exchange-rate fluctuation is guaranteed to be on $[m, M]$. Note that the rate does not necessarily reach either $m$ or $M$. The trader is allowed to exchange an arbitrary amount of dollars to yen on each transaction but not allowed to get dollars back. We also assume that the cost of sampling the exchange rate and the transaction fees are negligible. There is a sudden end of the game at which the trader is obliged to exchange all the remaining dollars at the possible lowest rate $m$. The trader is not informed in advance when the game ends.

\footnotetext{
${ }^{1}$ This work was partially supported by KAKENHI (16092216, 19700015, and 19740059).
} 


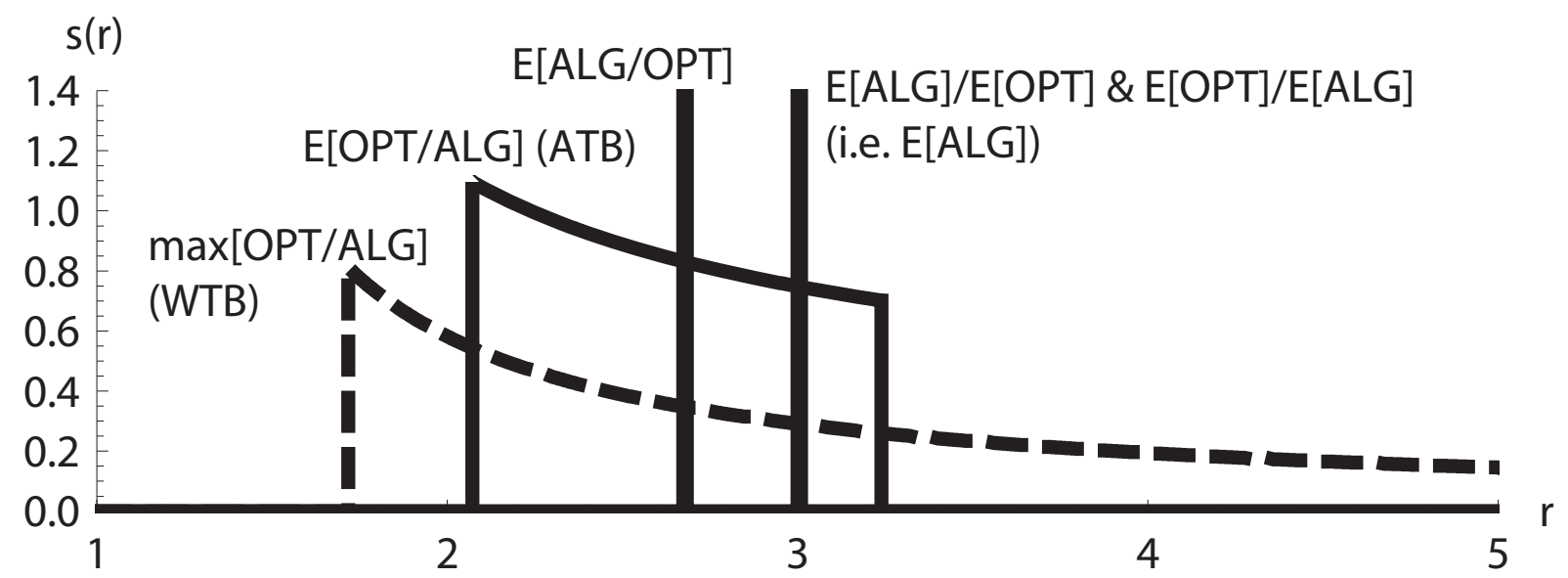

Figure 1: Optimal exchange strategies according to five different measures: The amount of dollars $s(r) d r$ to be exchanged when the exchange rate reaches $r$ for the first time.

El-Yaniv et al. [EFKT01] presented the worst-case threat-based strategy WTB: (i) If the current exchange rate $q$ is the highest thus far, then the trader should exchange $\int_{q_{0}}^{q} s_{w}(r) d r$ dollars, where $s_{w}(r)=\frac{1}{c_{w}(r-m)}$ for $m c_{w} \leq r \leq M$ and zero elsewhere, and $q_{0}$ is the highest rate on the previous transaction. (ii) Otherwise, the trader should not exchange. $c_{w}$ is a constant determined by the equation $c=\ln \frac{M-m}{m(c-1)}$. Denoting the returns of an online and an optimal offline strategies by ALG and OPT, respectively, WTB minimizes the worst-case competitive ratio max $\left[\frac{\mathrm{OPT}}{\mathrm{ALG}}\right]$ (and maximizes $\min \left[\frac{\mathrm{ALG}}{\mathrm{OPT}}\right]$ ). They also proved that randomization does not help, i.e., the ratio cannot be improved even if the strategy is randomized. Thus there does not seem any room for further improvement.

However, it is also true that worst-case analysis is often criticized as too pessimistic, and average-case analysis has always been an interesting research target. For online algorithms as well, several objective functions as presented above have recently appeared in the literature [FI05, PS06, Bec04, SSS06, NR08, GGLS08]. Our current problem involves a lot of human activities; it seems especially interesting to make an analysis under proper input distributions.

\subsection{Our Contribution}

Our goal in this paper is average-case competitive analyses for one-way trading under several input distributions and performance measures. Our first contribution is to show that the optimal strategy becomes significantly different depending on the performance measure, such as $E\left[\frac{\mathrm{OPT}}{\mathrm{ALG}}\right], \frac{E[\mathrm{OPT}]}{E[\mathrm{ALG}]}, E\left[\frac{\mathrm{ALG}}{\mathrm{OPT}}\right]$, or $\frac{E[\mathrm{ALG}]}{E[\mathrm{OPT}]}$. Figure 1 illustrates the optimal amount of dollars to be exchanged when the exchange rate reaches $r$ for the first time, under the assumption that the maximum rate of a game is uniformly distributed from between one and five. For comparison, $s_{w}$ of the strategy WTB, independent of the distribution, is also drawn with a dashed line. (A) One can easily distinguish the difference between $E\left[\frac{\mathrm{OPT}}{\mathrm{ALG}}\right]$ and the others: Only the strategy that minimizes $E\left[\frac{\mathrm{OPT}}{\mathrm{ALG}}\right]$, which we call the average-case threat-based strategy (ATB), exchanges little by little on a certain rate range. In addition, ATB behaves differently also from WTB: Whereas WTB waits for the possible maximum rate with keeping some dollars, ATB completes the transaction by the time the rate grows up to 3.24. (B) Note that the minimization of $E\left[\frac{\mathrm{OPT}}{\mathrm{ALG}}\right]$ and the maximization of $E\left[\frac{\mathrm{ALG}}{\mathrm{OPT}}\right]$ lead to different results. Recall that for worst-case analysis it does not make sense to change max and min with taking reciprocal. Unlike ATB, the 
strategy that maximizes $E\left[\frac{\mathrm{ALG}}{\mathrm{OPT}}\right]$ is to exchange all at once at rate 2.67 , which is drawn as a delta function in Figure 1. (C) The minimization of $\frac{E[\mathrm{OPT}]}{E[\mathrm{ALG}]}$ and the maximization of $\frac{E[\mathrm{ALG}]}{E[\mathrm{OPT}]}$ are essentially the same problem, i.e., the maximization of $E[\mathrm{ALG}]$, since $E[\mathrm{OPT}]$ is independent of the online strategy. The result is to exchange all at once at rate 3.00. We will prove that for an arbitrary distribution both the maximization of $E\left[\frac{\mathrm{ALG}}{\mathrm{OPT}}\right]$ and that of $E[\mathrm{ALG}]$ result in such one-time transaction. It is also shown that for an IFR distribution (for the definition please refer to Section 5), the exchange timing for $E\left[\frac{\mathrm{ALG}}{\mathrm{OPT}}\right]$ is earlier than that for $E[\mathrm{ALG}]$.

We derive the performance of ATB and others all in closed form. This mathematically nontrivial analysis itself is another important contribution of this paper. Firstly, for one-way trading it is required to handle a function that describes a strategy, i.e. when and how much to exchange, which obviously makes the analysis involved. Secondly, unlike other average-case measures, this objective function is nonlinear: The return of an online strategy appears in the denominator. El-Yaniv et al. gave some results on $E[A L G]$, which are derived implicitly based on the linearity [EFKT01]. We constructively analyze $E\left[\frac{\mathrm{OPT}}{\mathrm{ALG}}\right]$ with the help of the calculus of variations. More specifically, a subproblem over a smaller solution space is first solved. Then we confirm the sufficient condition for the original problem.

\subsection{Previous Work}

Although the worst-case analysis introduced by Sleator and Tarjan [ST85] has provided us with beautiful results in online computation, its limitation has also been revealed: (a) The evaluation is often too pessimistic and therefore resulting optimal strategies seem far from practical ones, for instance the optimal strategy for one-way trading is to wait for the possible maximum rate with some dollars left. (b) In some cases the worst-case competitive ratio cannot tell the performance difference properly, e.g. all of the algorithms LRU, FWF, and FIFO for paging have the same worst-case competitive ratio despite the clear difference in empirical performance [BE98]. For supplementing these weak points, average-case analysis using $E\left[\frac{\mathrm{ALG}}{\mathrm{OPT}}\right]$ and so on has been recently proposed. Note that the expectation $E[\cdot]$ is taken with respect to the input distribution.

To overcome the weak point (a), [FI05] first employed $E\left[\frac{\mathrm{ALG}}{\mathrm{OPT}}\right]$ as a criterion of optimality for designing strategies for the ski-rental problem and presented a family of optimal online strategies which is different from that for worst-case analysis. The significant difference in analysis between the ski-rent problem and one-way trading is as follows: Whereas a strategy for the ski-rental problem is represented by a single real number which specifies when to buy skis, for one-way trading, as mentioned, we need to determine a function for trading.

Other than the model that an input is drawn from a distribution that is known by the online player in advance, several average-case models have been considered: Koutsoupias and Papadimitriou introduced the diffuse adversary model in which the online player knows the class of input distributions and the adversary attacks by choosing the worst distribution from the class [KP94]. In the smoothed competitive analysis [BLMS $\left.{ }^{+} 06\right]$, the input is probabilistically distributed around a parameter selected by the adversary.

As for the difficulty (b), paging and online bin packing have been intensively studied. Becchetti showed the difference in performance between FWF and LRU using $\frac{E[\mathrm{ALG}]}{E[\mathrm{OPT}}[\mathrm{Bec} 04]$. Shor studied the difference in the expected wasted spaces generated by the packing algorithms Best Fit and First Fit, whose worst-case competitive ratios are the same [Sho86]. Please see [FW98] for further results.

It seems itself of interest whether to take the expectation of ratios or the ratio of expectations. Panagiotou and Souza investigated the performance of LRU for different cache size and mentioned that in this case $E\left[\frac{\mathrm{ALG}}{\mathrm{OPT}}\right]$ measures more adequately than $\frac{E[\mathrm{ALG}]}{E[\mathrm{OPT}]}[\mathrm{PS} 06]$. Con- 
cerning bin packing, in contrast, $E\left[\frac{\mathrm{ALG}}{\mathrm{OPT}}\right]$ and $\frac{E[\mathrm{ALG}]}{E[\mathrm{OPT}]}$ make no difference as far as we consider the asymptotic ratio for a sufficiently long input sequence [NR08]. Garg et al. recently pointed out the difference between $E\left[\frac{\mathrm{ALG}}{\mathrm{OPT}}\right]$ and $\frac{E[\mathrm{ALG}]}{E[\mathrm{OPT}]}$ for the online Steiner tree problem: To obtain an upper bound of $E\left[\frac{\mathrm{ALG}}{\mathrm{OPT}}\right]$ requires a scenario-wise comparison independently of results for $\frac{E[\mathrm{ALG}]}{E[\mathrm{OPT}]}[\mathrm{GGLS} 08]$.

In stochastic scheduling the processing time of a job is assumed to be a random variable and therefore the performance of an algorithm has been measured based on average-case performance, usually $\frac{E[\mathrm{ALG}]}{E[\mathrm{OPT}]}[\mathrm{DLK} 82]$. For stochastic complete-time scheduling Scharbrodt et al. analyzed $E\left[\frac{\mathrm{ALG}}{\mathrm{OPT}}\right]$ and illustrated its validity [SSS06]. Combining the conventional stochastic scheduling and online optimization, Megow et al. proposed a stochastic online scheduling model in which a job arrives online and its processing time is drawn probabilistically [MUV06].

El-Yaniv et al. introduced one-way trading in the context of online computation in their conference paper [EFKT92]. They gave optimal strategies using worst-case analysis, as well as some bounds of the worst-case competitive ratio. Al-Binali provided an optimal strategy in a risk-reward framework in which the trader has an acceptable risk level and a forecast of the exchange-rate fluctuation [al-99]. Iwama and Yonezawa generalized this framework in that they introduced a below forecast and designed multi-phased strategies [IY99]. Chen et al. obtained the worst-case competitive ratio in explicit form under the assumption that the exchange rate approximately follows the geometric Brownian motion [CKLW01]. Lorenz et al. studied the problem under the setting that at each point of time the trader chooses whether to convert a fixed fraction of the initial wealth or to do nothing [LPS07]. Other models, including two-way trading and portfolio selection, are mentioned in [BE98].

\section{Preliminaries for the Calculus}

First of all, we shall explain the motivation to apply Lebesgue integration in this paper. The most important objective of our work is to solve the minimization of $E\left[\frac{\mathrm{OPT}}{\mathrm{ALG}}\right]$. Unfortunately, it seemed rather difficult to solve this problem directly. We thus began with some numerical experiments to find an optimal over all finite sequences each of which describes a strategy. Observing the results, we conjectured that a family of discontinuous functions achieves the minimum. Therefore, we choose the function space $L^{1}[m, M]$ as a feasible set in order to deal with such discontinuous functions comprehensively. $L^{1}[m, M]$ denotes the whole set of Lebesgue integrable real-valued functions $f$ on $[m, M]$ for which $\int_{m}^{M}|f(x)| d x<\infty$ (see e.g. [Roy88]). We also use the notation of $C[m, M]$ for the whole set of continuous real-valued functions on $[m, M]$.

We discuss Lebesgue-Stieltjes integration somewhere as needed. It is enough just to care the treatment of discontinuous points as below. Let $F$ be a non-decreasing function on $[a, b]$ that is discontinuous at $c \in[a, b]$ and differentiable elsewhere, and $g$ be a continuous function on $[a, b]$. Denoting the derivative of $F$ by $f$, we calculate

$$
\int_{a}^{b} g(x) d F(x)=\int_{a}^{c} g(x) f(x) d x+\int_{c}^{b} g(x) f(x) d x+g(c)(F(c+)-F(c-)),
$$

where $F(c+)$ and $F(c-)$ stand for $\lim _{\epsilon \rightarrow+0} F(c+\epsilon)$ and $\lim _{\epsilon \rightarrow+0} F(c-\epsilon)$, respectively. We will use these notations throughout this paper.

Once again, our goal is to find a function that minimizes the functional $E\left[\frac{\mathrm{OPT}}{\mathrm{ALG}}\right]$. Let us begin by describing a necessary condition for an extremum. The Gâteaux differential [Lue69] of a functional $J: L^{1}[m, M] \rightarrow \mathbb{R}$ at $f$ with increment $v \in L^{1}[m, M]$ is defined as

$$
D J(f) v:=\lim _{\epsilon \rightarrow 0} \frac{1}{\epsilon}\{J(f+\epsilon v)-J(f)\} .
$$


A necessary condition for $J$ to have an extremum at $\bar{f} \in L^{1}[m, M]$ is that

$$
D J(\bar{f}) v=0
$$

holds for all $v \in L^{1}[m, M]$. We usually proceed with our argument as follows: Suppose that $D J(\bar{f}) v$ is written as a form of $\int_{m}^{M} g(\bar{f}, x) v(x) d x$. Then, the fundamental lemma in variational calculus implies that $g(\bar{f}, x)=0$ for all $x \in[m, M]$ (see e.g. [Lue69]).

Besides, we introduce the Kuhn-Tucker condition for the optimization of a functional with inequality constraints. What one should note is, differently from the optimization over a finitedimensional vector space, the treatment of constraints over a real-valued domain. Suppose that the constraint " $f(x) \geq 0$ for all $x \in[m, M]$ " is added to the above example on $J$. Then, the Kuhn-Tucker condition [Lue69] (i.e. a necessary condition) for the problem to have an extremum at $\bar{f}$ is that there exists $\mu \in L^{\infty}[m, M]$ such that

$$
\begin{gathered}
\mu(x) \leq 0 \text { almost everywhere on }[m, M], \\
\qquad \int_{m}^{M} \mu(x) \bar{f}(x) d x=0,
\end{gathered}
$$

and

$$
D J(\bar{f}) v+\int_{m}^{M} \mu(x) v(x) d x=0
$$

for all $v \in L^{1}[m, M]$. ( $\mu$ can be regarded as a Lagrange Multiplier for a finite-dimensional case.) As far as this paper is concerned, it suffices to consider $\mu \in B[m, M] \subset L^{\infty}[m, M]$ such that instead of (1),

$$
\mu(x) \leq 0 \text { for all } x \in[m, M]
$$

which is a stronger condition. $B[m, M]$ denotes the whole set of functions $\mu$ on $[m, M]$ for which there exists $K \in \mathbb{R}$ such that $|\mu(x)|<K$ for all $x \in[m, M]$. We thus omit to explain $L^{\infty}$ and "almost everywhere", which are not directly necessary in the later arguments. Please refer to [Roy88] for further study on function spaces and measure theory.

\section{Worst-Case Threat-Based Strategy}

Recall that in our model the trader knows the possible range $[m, M]$ of the exchange-rate fluctuation and does not know when the game ends. (In [EFKT01] this model is referred to as Variant 2.) For convenience of calculus we assume that the exchange rate $q(t)$ is piecewise continuous with time $t \geq 0$. The trader can exchange $\int_{t}^{t+\Delta t} d \tilde{S}(t)$ dollars into $\int_{t}^{t+\Delta t} q(t) d \tilde{S}(t)$ yen in an arbitrarily small time interval $\Delta t$. Thus the strategy of the trader is represented by a function $\tilde{S}:[0, \infty) \rightarrow[0,1]$. The rule of one-way transaction forces $\tilde{S}$ to be a non-decreasing function. Suppose that the game is over at time $\tau$. Then the trader has to exchange the remaining dollars at the minimum rate $m$. The total return is written as

$$
\operatorname{ALG}_{\tilde{S}}(\tau)=\int_{0}^{\tau} q(t) d \tilde{S}(t)+m\left(1-\int_{0}^{\tau} d \tilde{S}(t)\right)=m+\int_{0}^{\tau}(q(t)-m) d \tilde{S}(t)
$$

On the other hand, the optimal offline strategy will exchange the whole one dollar at the highest rate throughout the game and therefore its return is $\mathrm{OPT}(\tau)=\max _{0 \leq x \leq \tau} q(x)$.

El-Yaniv et al. stated that it is sufficient to consider strategies that exchange only when the exchange rate is the highest so far [EFKT01]. Let $\bar{q}(t)$ denote $\max _{0 \leq x \leq t} q(x)$. Such a strategy exchanges $\int_{\bar{q}(t)}^{q(t+\Delta t)} d S(r)$ dollars if $q(t+\Delta t)>\bar{q}(t)$ and does not exchange otherwise, where 
$S:[m, M] \rightarrow[0,1]$ is a non-decreasing function. We distinguish between these two categories of strategies by denoting $\tilde{S}$ and $S$; strategies with respect to time and those with respect to the highest exchange rate. We also describe a strategy with respect to the exchange rate by $s(r):=\frac{d S(r)}{d r}$ if the derivative of $S$ exists. Suppose that the highest exchange rate of the game is $p$. Without loss of generality, we assume $q(0)=m$. The return is obtained as

$$
\mathrm{ALG}_{S}=\int_{m}^{p} r d S(r)+m\left(1-\int_{m}^{p} d S(r)\right)=m+\int_{m}^{p}(r-m) d S(r) .
$$

Obviously, the optimal offline strategy gains $\mathrm{OPT}=p$. We later denote $\mathrm{ALG}_{S}$ simply by ALG when $S$ is clear from the context. Here we present the (worst-case) threat-based strategy proposed by El-Yaniv et al. Let $c_{w}$ be the root of the equation $c=\ln \frac{M-m}{m(c-1)}$.

Strategy WTB Suppose that the exchange rate changes from $q(t)$ to $q(t+\Delta t)$. (i) If $q(t+$ $\Delta t)>\bar{q}(t)$ then exchange $\int_{\bar{q}(t)}^{q(t+\Delta t)} s_{w}(r) d r$ dollars, where

$$
s_{w}(r)= \begin{cases}\frac{1}{c_{w}(r-m)}, & m c_{w} \leq r \leq M ; \\ 0, & m \leq r<m c_{w}\end{cases}
$$

(ii) If $q(t+\Delta t) \leq \bar{q}(t)$ then do not exchange.

Theorem 1 ([EFKT01]). For any $q$, the strategy WTB minimizes the worst-case competitive ratio $\max _{\tau} \frac{O P T(\tau)}{A L G_{\tilde{S}}(\tau)}$ to $c_{w}$.

\section{Average-Case Threat-Based Strategy}

We consider the model in which the highest exchange rate until a game is over follows a probability distribution and the trader devises a strategy with help of the knowledge of it. A distribution is characterized by a cumulative distribution function $F:[m, M] \rightarrow[0,1]$ such that $F(p)$ is the probability that the highest exchange rate of the game is equal to or less than $p$. Throughout this paper, $E[\cdot]$ denotes the expectation with respect to $F$ unless otherwise specified as $E_{H}[\cdot]$ for a distribution $H$. We will seek an optimal online strategy that minimizes

$$
E\left[\frac{\mathrm{OPT}}{\mathrm{ALG}}\right]=\int_{m}^{M} \frac{p}{m+\int_{m}^{p}(r-m) d S(r)} d F(p)
$$

among those that exchange only when the exchange rate is the highest so far.

Lemma 2 claims that it suffices to consider such a family of strategies, as it is the case in the worst-case analysis. More specifically, if the highest exchange rate is drawn from a distribution, an optimal strategy with respect to the highest exchange rate is also optimal among those with respect to time which may exchange even not at the highest rates. As the same as in Section 3, let $q$ be a rate function of time and $\bar{q}(t):=\max _{0 \leq x \leq t} q(x)$. For a given $q$, let $D:=\{t: 0 \leq \exists x<t, q(x) \geq q(t)\}$, i.e., the set of time instant at which the current rate is not the highest so far. We begin with a lemma that any strategy with respect to time can be converted into one that exchanges only at the highest rates without reducing its gain. Please recall that a strategy with (without) a tilde denotes one with respect to time (the highest exchange rate, respectively).

Lemma 1. For any $q$ and any strategy $\tilde{S}_{0}$, there exists a strategy $\tilde{S}$ such that $S$ is constant on $D$ and $A L G_{\tilde{S}}(\tau) \geq A L G_{\tilde{S}_{0}}(\tau)$ for all $\tau \geq 0$. 
Proof. One can see that $D$ consists of a family of disjoint intervals, which are labeled as $D_{1}, D_{2}, \ldots, D_{l}$. Let us set $a_{i}=\inf D_{i}$ and $b_{i}=\sup D_{i}$ for all $1 \leq i \leq l$. For each $D_{i}$, one can choose $z_{i} \notin D$ such that $q\left(z_{i}\right) \geq \sup _{x \in D_{i}} q(x)$ and $z_{i} \leq a_{i}$. We compose $\tilde{S}_{1}, \tilde{S}_{2}, \ldots, \tilde{S}_{l}$ from $\tilde{S}_{0}$ by

$$
\tilde{S}_{i}(t)= \begin{cases}\tilde{S}_{i-1}(t)+\tilde{S}_{i-1}\left(b_{i}\right)-\tilde{S}_{i-1}\left(a_{i}-\right), & z_{i} \leq t<a_{i} \\ \tilde{S}_{i-1}\left(b_{i}\right), & a_{i} \leq t<b_{i} \\ \tilde{S}_{i-1}(t), & 0 \leq t<z_{i}, b_{i} \leq t\end{cases}
$$

for $1 \leq i \leq l$. It follows that

$$
\begin{aligned}
\lim _{\epsilon \rightarrow+0} \int_{a_{i}-\epsilon}^{b_{i}}(q(t)-m) d \tilde{S}_{i-1}(t) & \leq\left(\sup _{x \in D_{i}} q(x)-m\right) \cdot\left(\tilde{S}_{i-1}\left(b_{i}\right)-\tilde{S}_{i-1}\left(a_{i}-\right)\right) \\
& \leq\left(q\left(z_{i}\right)-m\right) \cdot\left(\tilde{S}_{i-1}\left(b_{i}\right)-\tilde{S}_{i-1}\left(a_{i}-\right)\right) \\
& =\lim _{\epsilon \rightarrow+0} \int_{z_{i}-\epsilon}^{z_{i}}(q(t)-m) d \tilde{S}_{i}(t) .
\end{aligned}
$$

Since each $z_{i}$ is a lower bound of $D_{i}$, we have for all $\tau \geq 0$,

$$
\int_{0}^{\tau}(q(t)-m) d \tilde{S}_{0}(t) \leq \int_{0}^{\tau}(q(t)-m) d \tilde{S}_{1}(t) \leq \cdots \leq \int_{0}^{\tau}(q(t)-m) d \tilde{S}_{l}(t) .
$$

By adding $m$ for each and rewriting $\tilde{S}_{l}$ as $\tilde{S}$, the proof is completed.

Given a distribution $F$ and a rate function $q$, consider a distribution $H:[0, \infty) \rightarrow[0,1]$ defined as $H(t):=F(\bar{q}(t))$ for all $t \geq 0$. Then, one can observe that $H(t)$ is the probability that the game ends by the time $t$, since $F(p)$ is the probability that (the highest exchange rate) $\leq p$. Indeed, $H$ increases while the exchange rate hits a new high, and remains constant otherwise.

Lemma 2. Suppose that there exists a strategy $S^{*}$ such that $E_{F}\left[O P T / A L G_{S^{*}}\right] \leq E_{F}\left[O P T / A L G_{S}\right]$ holds for all $S$. Then, $E_{H}\left[O P T(t) / A L G_{\tilde{S}^{*}}(t)\right] \leq$ $E_{H}\left[O P T(t) / A L G_{\tilde{S}}(t)\right]$ for all $\tilde{S}$, where $\tilde{S}^{*}(t):=S^{*}(\bar{q}(t))$ for all $t \geq 0$.

Proof. Assume that there exists $\tilde{S}_{0}$ such that

$$
E_{H}\left[\frac{\mathrm{OPT}(t)}{\mathrm{ALG}_{\tilde{S}^{*}}(t)}\right]>E_{H}\left[\frac{\mathrm{OPT}(t)}{\operatorname{ALG}_{\tilde{S}_{0}}(t)}\right] .
$$

Since $H$ and $\tilde{S}^{*}$ are constant on $D$ by definition, we have

$$
\begin{aligned}
E_{H}\left[\frac{\mathrm{OPT}(t)}{\operatorname{ALG}_{\tilde{S}^{*}}(t)}\right] & =\int_{[0, \infty) \backslash D} \frac{\mathrm{OPT}(t)}{\mathrm{ALG}_{\tilde{S}^{*}}(t)} d H(t) \\
& =\int_{[0, \infty) \backslash D} \frac{\mathrm{OPT}(t)}{\mathrm{ALG}_{\tilde{S}^{*}}(t)} d F(\bar{q}(t)) \\
& =\int_{m}^{M} \frac{\mathrm{OPT}}{\mathrm{ALG}_{S^{*}}} d F(p) \\
& =E_{F}\left[\frac{\left.\mathrm{OPT}^{\mathrm{ALG}_{S^{*}}}\right],}{}\right.
\end{aligned}
$$


where $p:=\bar{q}(t)$. On the other hand, Lemma 1 implies that there exists $\tilde{S}$ such that $\tilde{S}$ is constant on $D$ and

$$
E_{H}\left[\frac{\mathrm{OPT}(t)}{\operatorname{ALG}_{\tilde{S}_{0}}(t)}\right] \geq E_{H}\left[\frac{\mathrm{OPT}(t)}{\operatorname{ALG}_{\tilde{S}}(t)}\right] .
$$

Consider $S$ defined as $S(\bar{q}(t)):=\tilde{S}(t)$ for all $t \geq 0$. Arguing similarly to above we obtain

$$
E_{H}\left[\frac{\mathrm{OPT}(t)}{\mathrm{ALG}_{\tilde{S}}(t)}\right]=E_{F}\left[\frac{\mathrm{OPT}}{\mathrm{ALG}_{S}}\right] .
$$

Thus we have

$$
E_{F}\left[\frac{\mathrm{OPT}}{\mathrm{ALG}_{S^{*}}}\right]>E_{F}\left[\frac{\mathrm{OPT}}{\mathrm{ALG}_{S}}\right]
$$

which contradicts the optimality of $S^{*}$.

As a result of the analysis in Section 4.3 we obtain an optimal strategy, which we name the average-case threat-based strategy (ATB). This strategy advises to exchange dollars only during a certain exchange-rate range of $[\alpha, \beta] \subset[m, M]$. In what follows we assume that the distribution $F$ has a positive density function $f$.

Strategy ATB Suppose that the exchange rate changes from $q(t)$ to $q(t+\Delta t)$. (i) If $q(t+$ $\Delta t)>\bar{q}(t)$ then exchange $\int_{\bar{q}(t)}^{q(t+\Delta t)} s_{a}(r) d r$ dollars, where

$$
s_{a}(r)= \begin{cases}\frac{m}{(\alpha-m) \sqrt{\alpha f(\alpha)}}\left\{\frac{3 r-m}{2(r-m)} \sqrt{\frac{f(r)}{r}}+\frac{f^{\prime}(r)}{2} \sqrt{\frac{r}{f(r)}}\right\}, & \alpha \leq r \leq \beta \\ 0, & \text { otherwise. }\end{cases}
$$

(ii) If $q(t+\Delta t) \leq \bar{q}(t)$ then do not exchange. $\alpha$ and $\beta$ are constants given by

$$
\int_{\alpha}^{\beta} s_{a}(r) d r=1 \text { and } \beta(\beta-m) f(\beta)=\int_{\beta}^{M} p f(p) d p .
$$

Given $f$, one can have the value of $\beta$ from the second equation in (3). The value of $\alpha$ is determined by the first equation in (3) after substituting $s_{a}$ with $\alpha$ unknown. The optimality of ATB requires the Condition $(C)$ which appears in Lemma 4. A simple sufficient condition for $(C)$ is the following $(\widetilde{C})$.

$(\widetilde{C}) f$ is non-decreasing on $[m, \beta]$ and $(r-m)^{2} r f(r) \geq(\beta-m)^{2} \beta f(\beta)$ holds for all $r \in[\beta, M]$.

Examples of ATB for several distributions will follow shortly before showing the optimality.

\subsection{Uniform Distribution}

Consider the probability density function $f(p)=\frac{1}{M-m}$, which means that the highest rate $p$ is uniformly distributed on the range $[m, M]$. The optimal online strategy ATB is then represented by

$$
s_{a}(r)= \begin{cases}\frac{m}{(\alpha-m) \sqrt{\alpha}} \cdot \frac{3 r-m}{2(r-m) \sqrt{r}}, & \alpha \leq r \leq \beta \\ 0, & \text { otherwise. }\end{cases}
$$

We derive $\beta=\frac{1}{3}\left(m+\sqrt{m^{2}+3 M^{2}}\right)<m+\frac{2}{3}(M-m)$ from (3). (The other root is negative and therefore infeasible.) One can easily confirm the Condition $(\widetilde{C})$. Note that the uniform 


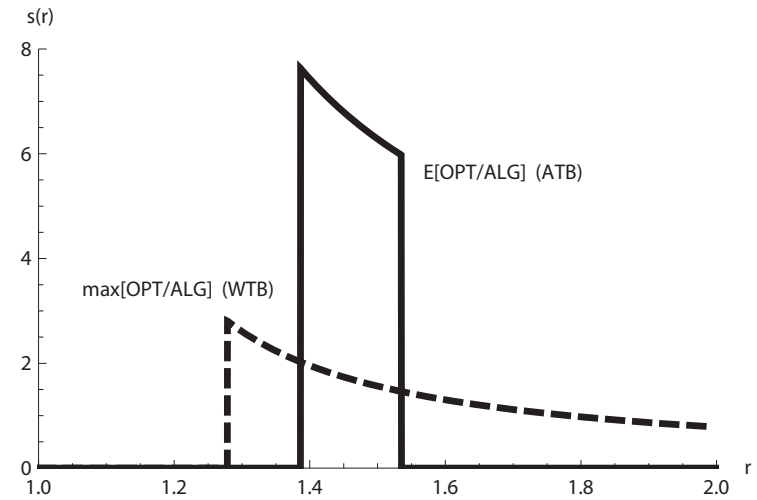

Figure 2: ATB and WTB for a uniform distribution.

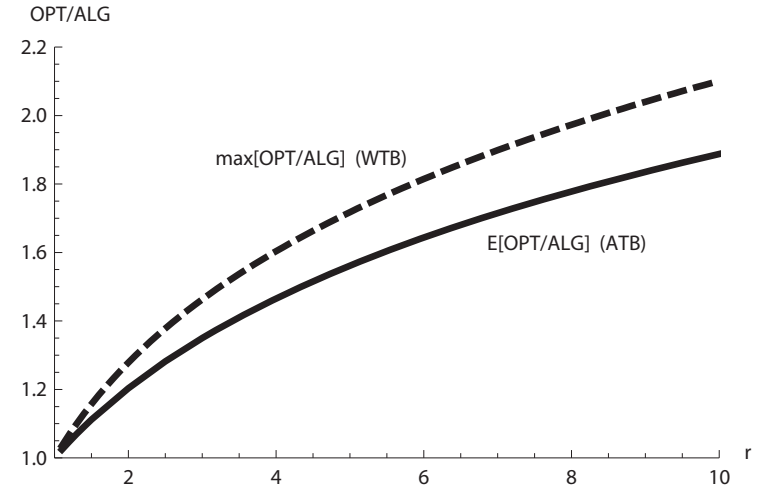

Figure 3: Performance ratios of ATB and WTB for a uniform distribution $(M / m=1$ to 10$)$.

distribution means that we have a completely even chance of the highest exchange rate in the range. This allows us to concentrate on rather narrow range for trade, which is obviously impossible if we assume the worst-case adversary. Actually, the above inequality implies that ATB completes the conversion before the rate reaches $66 \%$ of the range $[m, M]$. Figure 2 illustrates the case of $m=1$ and $M=2$. When ATB finishes the entire transaction, WTB still retains $48 \%$ of the initial asset. Moreover, WTB waits with some dollars left until the exchange rate finally reaches $M$, no matter how scarcely the luck happens. The advantage of ATB appears in the performance measure: By exchanging dollars intensively on a narrow range of $[1.38,1.53], E\left[\frac{\mathrm{OPT}}{\mathrm{ALG}}\right]$ is improved down to 1.20 from $1.29\left(=c_{w}=E\left[\frac{\mathrm{OPT}}{\mathrm{WTB}}\right]\right)$. For the setting of $m=1$ and $M=10$, the improvement is 1.88 from 2.10 by making transaction on the range $[2.8,6.1]$. Figure 3 illustrates $E\left[\frac{\mathrm{OPT}}{\mathrm{ALG}}\right]$ and $\max \left[\frac{\mathrm{OPT}}{\mathrm{ALG}}\right]$ for $M / m=1$ to 10 .

\subsection{Extreme Value Distribution}

If random variables are independent and identically distributed then the maxima under some affine transformation follow the generalized extreme value distribution [Ger89, EKM97]

$$
f(p)=\frac{1}{\sigma}\left(1+k \frac{p-\mu}{\sigma}\right)^{-1-\frac{1}{k}} \exp \left(-\left(1+k \frac{p-\mu}{\sigma}\right)^{-\frac{1}{k}}\right) .
$$

Apparently, this distribution provides the trader with useful information on the exchange rate. Consequently, the degree of improvement is outstanding compared with that of the uniform distribution. Again, let $m=1$ and $M=2$. Recall that the value of $c_{w}$ is 1.29 previously. (i) Weibull distribution, say, $k=-0.5, \sigma=0.12, \mu=1.77$ (see Figure 4): The density function has a peak at 1.84 and a long tail to the left. That is, the trader is informed that the rate is in an upward trend. ATB exchanges on the range $[1.59,1.66]$ and $E\left[\frac{\mathrm{OPT}}{\mathrm{ALG}}\right]$ is reduced down to 1.15 . This setting satisfies the Condition $(\widetilde{C})$. (ii) Fréchet distribution, say, $k=0.5, \sigma=0.2, \mu=1.39$ (see Figure 5): This setting yields a right-tailed distribution whose peak is at 1.32. The trader anticipates that the rate does not grow so much. ATB converts dollars on the range $[1.27,1.34]$ and $E\left[\frac{\mathrm{OPT}}{\mathrm{ALG}}\right]$ becomes 1.19. One can confirm the Condition $(C)$. 


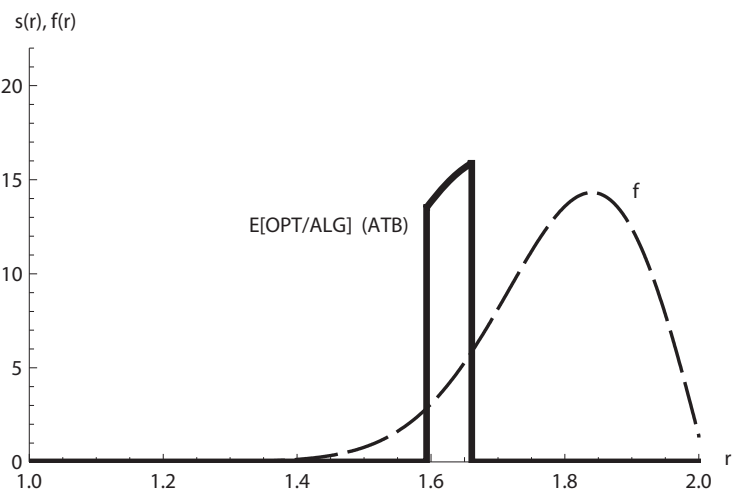

Figure 4: ATB for a Weibull distribution.

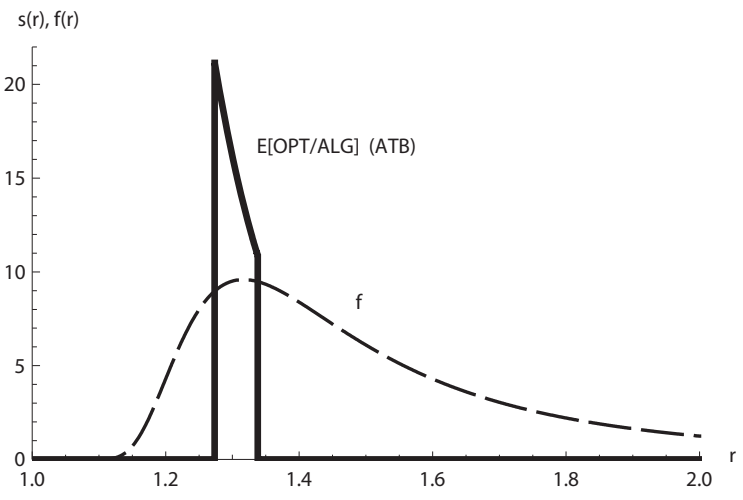

Figure 5: ATB for a Fréchet distribution.

\subsection{Proof of Optimality}

The proof of the optimality involves the calculus of variations. The minimization of $E\left[\frac{\mathrm{OPT}}{\mathrm{ALG}}\right]$ can be written as:

$$
\begin{aligned}
& (\mathcal{P}) \text { minimize } J(s):=\int_{m}^{M} \frac{p d F(p)}{m+\int_{m}^{p}(r-m) s(r) d r} \\
& \text { subject to } \int_{m}^{M} s(r) d r=1, s(r) \geq 0, s \in L^{1}[m, M] .
\end{aligned}
$$

Outline of the proof We first provide a sufficient condition for global optimality for $(\mathcal{P})$. Next we consider a subproblem $\left(\mathcal{P}_{0}\right)$ over a smaller solution space. Starting from a necessary condition for $\left(\mathcal{P}_{0}\right)$, we derive a local optimal solution. Finally, it is confirmed that the obtained solution satisfies the sufficient condition for the original problem $(\mathcal{P})$.

Lemma 3. Suppose that there exist feasible $\bar{s} \in L^{1}[m, M], \lambda \in \mathbb{R}$ and $\mu \in B[m, M]$ such that $\mu(r) \leq 0$ for all $r \in[m, M], \int_{m}^{M} \mu(r) \bar{s}(r) d r=0$ and

$$
\int_{m}^{M} \frac{-p \int_{m}^{p}(r-m) v(r) d r}{\left(m+\int_{m}^{p}(r-m) \bar{s}(r) d r\right)^{2}} d F(p)+\int_{m}^{M} \lambda v(r) d r+\int_{m}^{M} \mu(r) v(r) d r=0
$$

for all $v \in L^{1}[m, M]$. Then $\bar{s}$ is a unique solution to the problem $(\mathcal{P})$.

Proof. The objective function is Gâteaux differentiable on the feasible set with the derivative $D J(s)$ given by

$$
D J(s) v=\int_{m}^{M} \frac{-p \int_{m}^{p}(r-m) v(r) d r}{\left(m+\int_{m}^{p}(r-m) \bar{s}(r) d r\right)^{2}} d F(p)
$$

for each $v \in L^{1}[m, M]$. Since $\frac{1}{1+x}$ is strictly convex on $[0, \infty)$, we can show that $J$ is strictly convex on the convex feasible set. Indeed, for any two different strategies $s_{1}$ and $s_{2}$ in the feasible set and any $0<\gamma<1, J\left(\gamma s_{1}+(1-\gamma) s_{2}\right)<\gamma J\left(s_{1}\right)+(1-\gamma) J\left(s_{2}\right)$ holds. Hence, it follows that for each feasible $s, J(s) \geq J(\bar{s})+D J(\bar{s})(s-\bar{s})$. Also, $\int_{m}^{M} s(r) d r=1$ and $\int_{m}^{M} \mu(r) s(r) d r \leq 0$, 
since $\mu(r) \leq 0$ for all $r \in[m, M]$ as assumed. Then we have for each feasible $s$

$$
\begin{aligned}
J(s) & \geq J(s)+\lambda\left(\int_{m}^{M} s(r) d r-1\right)+\int_{m}^{M} \mu(r) s(r) d r \\
& \geq J(\bar{s})+D J(\bar{s})(s-\bar{s})+\lambda \int_{m}^{M}(s(r)-\bar{s}(r)) d r+\int_{m}^{M} \mu(r)(s(r)-\bar{s}(r)) d r \\
& =J(\bar{s})+(D J(\bar{s})+\lambda+\mu)(s-\bar{s}) \\
& =J(\bar{s}) .
\end{aligned}
$$

Therefore $\bar{s}$ is a minimizer of the problem $(\mathcal{P})$. Note that $\int_{m}^{M} \bar{s}(r) d r=1$ and $\int_{m}^{M} \mu(r) \bar{s}(r) d r=0$, and that we derive the last equality by applying (4).

Suppose $s_{0}$ also attains the minimum. Then the equalities hold in each relation above. Thus $\int_{m}^{M} \mu(r) s_{0}(r) d r=0$ and the strict convexity of $J$ ensures that $s_{0}=\bar{s}$.

Note In Lemma 3, it is mathematically more natural to consider $L^{\infty}[m, M]$ that is the dual space of $L^{1}[m, M]$ than $B[m, M]$, which requires the knowledge of functional analysis. The condition in this lemma then corresponds to the Kuhn-Tucker condition [Lue69]. To consider $B[m, M] \subset L^{\infty}[m, M]$ is sufficient for our arguments and therefore we do so.

Lemma 4. The Condition $(\widetilde{C})$ implies the following condition $(C)$.

$(C)$

$$
\begin{array}{rrr}
\alpha(\alpha-r)(\alpha-m) f(\alpha)-(r-m) \int_{r}^{\alpha} p f(p) d p \geq 0, & \forall r \in[m, \alpha] ; \\
(3 r-m) f(r)+(r-m) r f^{\prime}(r)>0, & \forall r \in(\alpha, \beta) ; \\
\beta(\beta-m)^{2} f(\beta)-(r-m) \int_{r}^{M} p f(p) d p \geq 0, & \forall r \in[\beta, M] .
\end{array}
$$

Proof. The Condition $(\widetilde{C})$ implies that $f^{\prime}(r) \geq 0$ for all $r \in[m, \beta]$. Therefore the second inequality straightforwardly holds. Let $g(r)=\alpha(\alpha-r)(\alpha-m) f(\alpha)-(r-m) \int_{r}^{\alpha} p f(p) d p$. By differentiating we have $g^{\prime}(r)=-\alpha(\alpha-m) f(\alpha)+r(r-m) f(r)-\int_{r}^{\alpha} p f(p) d p$. Again, $g^{\prime \prime}(r)=$ $(3 r-m) f(r)+(r-m) r f^{\prime}(r)$, which is positive. Together with $g^{\prime}(\alpha)=0$, it follows that $g^{\prime}(r) \geq 0$ for all $r \in[m, \alpha]$. Since $g(\alpha)=0$, we obtain the first inequality. By applying $r f(r) \geq(\beta-m)^{2} \beta f(\beta) /(r-m)^{2}$, we have $\beta(\beta-m)^{2} f(\beta)-(r-m) \int_{r}^{M} p f(p) d p \geq(r-m)(\beta-$ $m)^{2} \beta f(\beta) /(M-m)>0$ for all $r \in[\beta, M]$, which is sufficient for the third inequality.

Theorem 2. Suppose that a distribution $F$ has a density function $f$ which is positive, continuously differentiable, and satisfies the Condition $(C)$. Then the unique minimizer of the problem $(\mathcal{P})$ is $s_{a}$ in (2). The constants are given by (3).

Proof. First, we narrow the candidates of solutions to functions which are nonnegative and continuous on a subinterval of $[m, M]$ and zero elsewhere. Then the problem finding a minimizer 
from such family of functions has the following form:

$$
\begin{aligned}
&\left(\mathcal{P}_{0}\right) \text { minimize } J_{0}(s, \alpha, \beta) \\
&:=\int_{m}^{\alpha} \frac{p f(p)}{m} d p+\int_{\alpha}^{\beta} \frac{p f(p) d p}{m+\int_{\alpha}^{p}(r-m) s(r) d r} \\
&+\int_{\beta}^{M} \frac{p f(p) d p}{m+\int_{\alpha}^{\beta}(r-m) s(r) d r} ; \\
& \text { subject to } G(s, \alpha, \beta):=\int_{\alpha}^{\beta} s(r) d r=1, s(r) \geq 0, m<\alpha<\beta<M, \\
& s \in C[m, M] .
\end{aligned}
$$

Observe the problem $\left(\mathcal{P}_{0}\right)$ is no longer a convex program. Thus we find a function $\bar{s}$ which satisfies a necessary condition for local optimality in $\left(\mathcal{P}_{0}\right)$. Then we will confirm the condition in Lemma 3 by explicitly giving a constant $\lambda$ and a function $\mu$ there.

Suppose $(\bar{s}, \alpha, \beta)$ is a local optimal of $\left(\mathcal{P}_{0}\right)$ and $\bar{s}$ is positive. Then there exists $\lambda \in \mathbb{R}$ such that

$$
D J_{0}(\bar{s}, \alpha, \beta)(v, \xi, \eta)+\lambda D G(\bar{s}, \alpha, \beta)(v, \xi, \eta)=0
$$

for all $\xi, \eta \in \mathbb{R}, v \in C[m, M]$. Here

$$
\begin{aligned}
& D J_{0}(\bar{s}, \alpha, \beta)(v, \xi, \eta) \\
&=\left[\left\{\int_{\alpha}^{\beta} \frac{(\alpha-m) \bar{s}(\alpha) p f(p) d p}{\left(m+\int_{\alpha}^{p}(r-m) \bar{s}(r) d r\right)^{2}}+\frac{(\alpha-m) \bar{s}(\alpha) \int_{\beta}^{M} p f(p) d p}{\left(m+\int_{\alpha}^{\beta}(t-m) \bar{s}(t) d t\right)^{2}}\right\}\right] \\
&+\left\{\frac{(\beta-m) \int_{\beta}^{M} p f(p) d p}{\left(m+\int_{\alpha}^{\beta}(r-m) \bar{s}(r) d r\right)^{2}} \bar{s}(\beta)\right\} \eta+\int_{\alpha}^{\beta} \frac{-p f(p) \int_{\alpha}^{p}(r-m) v(r) d r}{\left(m+\int_{\alpha}^{p}(r-m) \bar{s}(r) d r\right)^{2}} d p \\
&+\int_{\beta}^{M} \frac{-p f(p) \int_{\alpha}^{\beta}(r-m) v(r) d r}{\left(m+\int_{\alpha}^{\beta}(r-m) \bar{s}(r) d r\right)^{2}} d p
\end{aligned}
$$

and

$$
D G(\bar{s}, \alpha, \beta)(v, \xi, \eta)=\bar{s}(\beta) \eta-\bar{s}(\alpha) \xi+\int_{\alpha}^{\beta} v(p) d p .
$$

By substituting $\xi=\eta=0$ in (5), we have

$$
\int_{\alpha}^{\beta} \frac{-p f(p) \int_{\alpha}^{p}(r-m) v(r) d r}{\left(m+\int_{\alpha}^{p}(t-m) \bar{s}(t) d t\right)^{2}} d p+A \cdot \int_{\alpha}^{\beta}(r-m) v(r) d r+\lambda \int_{\alpha}^{\beta} v(p) d p=0,
$$

where

$$
A=\frac{-\int_{\beta}^{M} p f(p) d p}{\left(m+\int_{\alpha}^{\beta}(t-m) \bar{s}(t) d r\right)^{2}}
$$

We change the order of the integration of the first term and replace $p$ with $r$ in the third term. Then we obtain

$$
\int_{\alpha}^{\beta} v(r)\left\{(r-m)\left(\int_{r}^{\beta} \frac{-p f(p) d p}{\left(m+\int_{\alpha}^{p}(t-m) \bar{s}(t) d t\right)^{2}}+A\right)+\lambda\right\} d r=0 .
$$


The fundamental lemma in variational calculus states that if the equation holds for every $v \in$ $C[m, M]$, then

$$
(r-m)\left(\int_{r}^{\beta} \frac{-p f(p) d p}{\left(m+\int_{\alpha}^{p}(t-m) \bar{s}(t) d t\right)^{2}}+A\right)+\lambda=0
$$

for all $r \in[\alpha, \beta]$. By substituting $r=\beta$ and $r=\alpha$ in (6), we have respectively $(\alpha-m) B+(\alpha-$ $m) A+\lambda=0$ and $(\beta-m) A+\lambda=0$, where

$$
B=\int_{\alpha}^{\beta} \frac{-p f(p) d p}{\left(m+\int_{\alpha}^{p}(t-m) \bar{s}(t) d t\right)^{2}} .
$$

We also have

$$
\int_{r}^{\beta} \frac{-p f(p) d p}{\left(m+\int_{\alpha}^{p}(t-m) \bar{s}(t) d t\right)^{2}}+(r-m) \frac{r f(r)}{\left(m+\int_{\alpha}^{r}(t-m) \bar{s}(t) d t\right)^{2}}+A=0
$$

by differentiating (6) and hence

$$
B+\frac{(\alpha-m) \alpha f(\alpha)}{m^{2}}+A=0
$$

by letting $r=\alpha$. Thus we obtain

$$
\lambda=\frac{(\alpha-m)^{2} \alpha f(\alpha)}{m^{2}}
$$

Then we eliminate the first term in (6) and (7) and hence

$$
(r-m)^{2} \frac{r f(r)}{\left(m+\int_{\alpha}^{r}(t-m) \bar{s}(t) d t\right)^{2}}-\lambda=0 .
$$

Thus we obtain

$$
m+\int_{\alpha}^{r}(t-m) \bar{s}(t) d t=\frac{1}{\sqrt{\lambda}}(r-m) \sqrt{r f(r)},
$$

since the left hand side should be positive. By differentiating we have

$$
\bar{s}(r)=\frac{1}{\sqrt{\lambda}}\left\{\frac{3 r-m}{2(r-m)} \sqrt{\frac{f(r)}{r}}+\frac{f^{\prime}(r)}{2} \sqrt{\frac{r}{f(r)}}\right\} .
$$

The second inequality in the Condition $(C)$ guarantees its feasibility. Now let us determine $\alpha$ and $\beta$. By substituting $\beta$ for $r$ in (7), we have

$$
\beta(\beta-m) f(\beta)=\int_{\beta}^{M} p f(p) d p
$$

which is the condition for $\beta$. Then, $\alpha$ is determined by $\int_{\alpha}^{\beta} \bar{s}(r) d r=1$ and (9). Therefore we have shown that $\bar{s}$ is equal to $s_{a}$.

To complete the proof, we use Lemma 3 to show the obtained function to be a minimizer of the original problem $(\mathcal{P})$. By substituting $\bar{s}$ for $s$ in (4) and changing the order of the 
integration, we have

$$
\begin{aligned}
\int_{m}^{\alpha} v(r) & \left\{\frac{1}{m^{2}}\left(-(r-m) \int_{r}^{\alpha} p f(p) d p+\alpha(\alpha-r)(\alpha-m) f(\alpha)\right)+\mu(r)\right\} d r \\
& +\int_{\alpha}^{\beta} v(r) \mu(r) d r+\int_{\beta}^{M} v(r) \\
& \cdot\left\{\frac{\lambda}{\beta(\beta-m)^{2} f(\beta)}\left(-(r-m) \int_{r}^{M} p f(p) d p+\beta(\beta-m)^{2} f(\beta)\right)+\mu(r)\right\} d r
\end{aligned}
$$

Suppose now the Condition $(C)$ is satisfied. Observing the first and third terms in the above equality, we can choose $\mu(r) \leq 0$ for all $r \in[m, \alpha]$ and $r \in[\beta, M]$, respectively, so that each term is equal to zero for any $v \in L^{1}[m, M]$. The second term also becomes zero by setting $\mu(r)=0$ for all $r \in(\alpha, \beta)$. We have thus confirmed that (4) is satisfied by obtained $\bar{s}, \lambda$, and $\mu$.

\subsection{Randomized Strategies}

The following result by El-Yaniv et al. shows that randomization of one-way trading strategies cannot improve $E_{G}$ [RALG] nor therefore the average-case performance measure such as $E_{F}\left[\frac{\mathrm{OPT}}{E_{G}[\mathrm{RALG}]}\right], E_{F}\left[\frac{E_{G}[\mathrm{RALG}]}{\mathrm{OPT}}\right]$, or $E_{F}\left[E_{G}[\mathrm{RALG}]\right]$, where $E_{G}[\mathrm{RALG}]$ denotes the expected return of a randomized strategy RALG. It is also mentioned that in one-way trading there is no essential difference between the two types in randomized strategies; a mixed strategy that is represented by a distribution over a class of deterministic strategies and a behavioral strategy that randomly determines the amount of dollars to be exchanged at every time.

Proposition 1 ([EFKT01]). Let $R A L G$ be any randomized strategy which may be a mixed or behavioral strategy. Then, there exists a deterministic strategy represented by $S$ such that $E_{G}[R A L G]=A L G_{S}$ for any scenario of the exchange-rate fluctuation. The reverse statement also holds true: For any deterministic strategy represented by $S$, there exists a randomized strategy RALG which satisfies the above equality.

\subsection{Minimax Theorem}

Online problems are usually handled as a game between an online player and an adversary. In our model the adversary selects a distribution and after that the online player determines the strategy. That is, the adversary cannot make any adaptive choice. Nevertheless, the next theorem states that even this type of adversary can attack the player as cruelly as the one who devises a strategy after observing the online player's decision.

\section{Theorem 3.}

$$
\max _{F} \min _{A L G} E\left[\frac{O P T}{A L G}\right]=\min _{A L G} \max _{F} E\left[\frac{O P T}{A L G}\right]=c_{w} .
$$

A remarkable point here is that we find a saddle point: $s_{w}(r)$ in (3), i.e., the strategy WTB and

$$
F_{w}(p)= \begin{cases}0, & p \in\left[m, m c_{w}\right) \\ \frac{p-m c_{w}}{c_{w}^{2}(p-m)}-\frac{c_{w}-1}{c_{w}^{2}} \ln \frac{m\left(c_{w}-1\right)}{p-m}, & p \in\left[m c_{w}, M\right) \\ 1, & p=M .\end{cases}
$$


In other words, $F_{w}$ represents the strongest adversary in our model.

One should note that one-way trading in this paper is an infinite game. For a finite game, Yao's principle that is derived from Loomis' lemma [MR95] provides a lower bound of the worstcase competitive ratio of randomized algorithms [BE98]. Indeed, if we narrow $F$ and RALG down to distributions over finite strategies such that RALG includes WTB, then an upper bound of can be given as follows: According to Loomis' lemma, $\max _{F} \min _{\mathrm{ALG}} E_{F}\left[\frac{\mathrm{OPT}}{\mathrm{ALG}}\right]=$ $\min _{G} \max _{p} E_{G}\left[\frac{\mathrm{OPT}}{\mathrm{RALG}}\right]$. Jensen's inequality implies that the right-hand-side equals

$\min _{G} \max _{p}\left[\frac{\mathrm{OPT}}{E_{G}[\mathrm{RALG}]}\right]=\min _{\mathrm{ALG}} \max _{p}\left[\frac{\mathrm{OPT}}{\mathrm{ALG}}\right]=c_{w}$, which is equivalent to the first equality in Theorem 3. However, our model treats uncountable strategy sets consisting of functions and distributions, for which Loomis' lemma does not hold. Theorem 3 is obtained independently with a constructive discussion. Another example that derives a special case of Yao's principle for an infinite game with a countable strategy set can be found in [BE98]. Also note that unlike other minimax theorems Theorem 3 holds for a noncompact strategy set.

Proof. Firstly, we prove $\min _{\mathrm{ALG}} \max _{F} E\left[\frac{\mathrm{OPT}}{\mathrm{ALG}}\right]=c_{w}$. We set the cumulative distribution function $F_{x}(p)=1$ for $p \in[x, M]$ and zero for $p \in[m, x)$ with $x$ chosen after the online strategy is revealed. This is equivalent to the adversary in a worst-case analysis. Therefore a lower bound is $c_{w}$. On the other hand, by substituting strategy $s_{w}$ of WTB the lower bound is achieved from Theorem 1.

Secondly, we show $\max _{F} \min _{\mathrm{ALG}} E\left[\frac{\mathrm{OPT}}{\mathrm{ALG}}\right]=c_{w}$. The sketch of the proof is as follows. We have already known that ATB minimizes for distributions satisfying the Condition $(C)$. We begin with formally applying ATB. Then we first maximize the objective functional over a particular subset of distributions. In fact, we consider a subspace including a given distribution $F_{0}$ as a feasible set. Then a parametric optimization problem is obtained with $F_{0}$ being the parameter. We find a parameter for which the optimal value coincides with $c_{w}$. Finally, we confirm that the obtained optimal solution is an optimal for the original problem, by applying Lemma 3.

Let us fix a distribution $F_{0}$ that has a density function $f_{0}$. We formally substitute $s_{a}$ of ATB for $s$. Then the function to be maximized is

$$
\int_{m}^{\alpha} \frac{p f_{0}(p)}{m} d p+\int_{\alpha}^{\beta} \frac{p f_{0}(p)}{\frac{1}{\sqrt{\lambda}}(p-m) \sqrt{p f_{0}(p)}} d p+\int_{\beta}^{M} \frac{p f_{0}(p)}{\frac{1}{\sqrt{\lambda}}(\beta-m) \sqrt{\beta f_{0}(\beta)}} d p .
$$

Note that we employed (8) instead of substituting $s_{a}$ directly and that $\lambda=\frac{(\alpha-m)^{2} \alpha f(\alpha)}{m^{2}}$. Then, for given $\alpha, \beta$, and $F_{0}$, consider $F$ that is equal to $F_{0}$ on $[m, \alpha]$ and smooth on $(\alpha, M)$. Then the maximization problem is

$$
\begin{aligned}
\text { maximize } & I(F):=\int_{m}^{\alpha} \frac{p}{m} d F(p)+\frac{m}{(\alpha-m) \sqrt{\alpha f(\alpha)}} \int_{\alpha}^{\beta} \frac{p f(p)}{(p-m) \sqrt{p f(p)}} d p \\
& +\frac{m}{(\alpha-m) \sqrt{\alpha f(\alpha)}} \int_{\beta}^{M} \frac{p f(p)}{(\beta-m) \sqrt{\beta f(\beta)}} d p \\
& +\frac{m M(F(M)-F(M-))}{(\alpha-m)(\beta-m) \sqrt{\alpha \beta f(\alpha) f(\beta)}} ; \\
\text { subject to } & \int_{m}^{M} d F(p)=1 \\
& d F(p)>0, p \in(\alpha, M] \\
& F(p)=F_{0}(p), p \in[m, \alpha],
\end{aligned}
$$


where $f$ is the function on $[\alpha, \beta]$ defined by

$$
f(p)= \begin{cases}F^{\prime}(\alpha+), & p=\alpha ; \\ F^{\prime}(p), & p \in(\alpha, \beta) \\ F^{\prime}(\beta-), & p=\beta .\end{cases}
$$

If the problem has an optimal $\bar{F}$, the Kuhn-Tucker theorem guarantees the existence of $\eta \in \mathbb{R}$ such that $\bar{F}$ also maximizes the functional

$$
I(F)+\eta\left(\int_{m}^{M} d F(p)-1\right)
$$

over all $F$ with $d F(p)>0$ and $F(p)=F_{0}(p)$ on $[m, \alpha]$. Let us consider a set $W$ of variations such that every $w \in W$ is nonzero on $(\alpha, \beta)$ and zero elsewhere. By considering the Gâteaux differential of the above functional, we have

$$
\int_{\alpha}^{\beta} w(p)\left\{\frac{m}{(\alpha-m) \sqrt{\alpha \bar{f}(\alpha)}} \frac{p}{2(p-m) \sqrt{p \bar{f}(p)}}+\eta\right\} d p=0
$$

for all $w \in W$, where $\bar{f}$ is defined similarly by $\bar{F}$. The fundamental lemma in variational calculus states that

$$
\frac{m}{(\alpha-m) \sqrt{\alpha \bar{f}(\alpha)}} \frac{p}{2(p-m) \sqrt{p \bar{f}(p)}}+\eta=0
$$

holds for all $p \in(\alpha, \beta)$. Then we obtain

$$
\bar{f}(p)=\frac{(\alpha-m)^{2} \alpha \bar{f}(\alpha)}{m^{2}} \frac{p}{4 \eta^{2}(p-m)^{2}}
$$

for all $p \in[\alpha, \beta]$. Letting $p=\alpha$ yields $\eta=-\alpha /(2 m)$, since $\eta$ should be nonpositive for satisfying the equation (11).

By applying (8) to (11) again, we have

$$
\frac{p}{m+\int_{\alpha}^{p}(r-m) s(r) d r}=-2 \eta=\frac{\alpha}{m} .
$$

Note that $s$ here is a strategy formally optimized with leaving $\alpha$ and $\beta$ to be original for $F_{0}$. Suppose that we have chosen $F_{0}$ such that $\alpha \leq m c_{w}$ and $\beta=M$. Now let us remind ourselves of WTB. If we substitute $s_{w}$ of WTB for $s$, then the left hand side of (12) is constant for $p \in\left[m c_{w}, M\right]$, which implies that $s_{w}$ is a candidate for $s$. In order for $s_{w}$ to be a solution, it is necessary to choose $F_{0}$ so that $\alpha / m=c_{w}$. In what follows, assume that we have chosen such $F_{0}$.

We next adjust the undetermined quantities $\bar{F}(M)-\bar{F}(M-)$ and $\bar{f}(\alpha)$ in the obtained distribution so that the optimality of WTB is consistent for this distribution. So, we set $\bar{F}(M)-\bar{F}(M-)$ to satisfy $M(M-m) \bar{f}(M)=\int_{\beta}^{M} p d \bar{F}(p)=M(\bar{F}(M)-\bar{F}(M-))$, which is obtained by applying slightly modified arguments in Theorem 2 to the case $\beta=M$. Since $\bar{F}$ is a probability distribution, we have

$$
\begin{aligned}
1= & \int_{m}^{\alpha} d \bar{F}(p)+\int_{\alpha}^{M} \bar{f}(p) d p+(\bar{F}(M)-\bar{F}(M-)) \\
= & \int_{m}^{\alpha} d F_{0}(p)+\frac{(\alpha-m)^{2} \bar{f}(\alpha)}{\alpha}\left\{\ln \frac{M-m}{\alpha-m}-\frac{m}{M-m}+\frac{m}{\alpha-m}\right\} \\
& +\frac{(\alpha-m)^{2} \bar{f}(\alpha)}{\alpha} \frac{M}{M-m} .
\end{aligned}
$$


Recall that $c_{w}=\ln \frac{M-m}{m\left(c_{w}-1\right)}$ and $\alpha=m c_{w}$. Then,

$$
\bar{f}(\alpha)=(1-K) \frac{\alpha}{(\alpha-m)^{2}}
$$

where $K=\int_{m}^{\alpha} d F_{0}(p)$. Eliminating $\bar{f}(\alpha)$ we have

$$
\begin{aligned}
\bar{f}(p) & =(1-K) \frac{m(\alpha-m)}{\alpha^{2}} \frac{p}{(p-m)^{2}} ; \\
\bar{F}(M)-\bar{F}(M-) & =(1-K) \frac{m(\alpha-m)}{\alpha^{2}} \frac{M}{M-m} .
\end{aligned}
$$

Now we set $F_{0}(p)=0$ on $[m, \alpha)$ and $F_{0}(\alpha)=K$. Then we obtain $I(\bar{F})=c_{w}$. Therefore if we can show that WTB is in fact an optimal when $K=0$, it completes the proof.

By applying Lemma 3 to the distribution above, it is sufficient to show that $s_{w}$ satisfies

$$
\begin{gathered}
\frac{m(\alpha-m)}{\alpha^{2}} \int_{\alpha}^{\beta} \frac{-p^{2} \int_{m}^{p}(r-m) v(r) d r}{(p-m)^{2}\left(m+\int_{\alpha}^{p}(r-m) s_{w}(r) d r\right)^{2}} d p \\
+\frac{m(\alpha-m)}{\alpha^{2}} \frac{M}{M-m} \frac{-M \int_{m}^{p}(r-m) v(r) d r}{\left(m+\int_{\alpha}^{M}(r-m) s_{w}(r) d r\right)^{2}} \\
\quad+\int_{m}^{M} \lambda v(r) d r+\int_{m}^{M} \mu(r) v(r) d r=0,
\end{gathered}
$$

for some $\lambda \in \mathbb{R}$ and $\mu \in B[m, M]$ with $\mu(r) \leq 0$ for all $r \in[m, M]$ and $\int_{m}^{M} \mu(r) s_{w}(r) d r=0$. The above equation is simplified as

$$
-\frac{1}{m} \int_{m}^{\alpha} v(r)(r-m) d r+\frac{-(\alpha-m)}{m} \int_{\alpha}^{M} v(r) d r+\int_{m}^{M} \lambda v(r) d r+\int_{m}^{M} \mu(r) v(r) d r=0 .
$$

Thus we can choose $\lambda=(\alpha-m) / m$ and

$$
\mu(r)= \begin{cases}-\frac{\alpha-r}{m}, & r \in[m, \alpha] \\ 0, & r \in(\alpha, M]\end{cases}
$$

so that the equality holds. Therefore WTB is an optimal for the obtained distribution and the proof is completed.

\section{Optimal Strategies for Other Measures}

In this section we analyze $E\left[\frac{\mathrm{ALG}}{\mathrm{OPT}}\right]$ and $E[\mathrm{ALG}]$. Note that $\frac{E[\mathrm{OPT}]}{E[\mathrm{ALG}]}$ and $\frac{E[\mathrm{ALG}]}{E[\mathrm{OPT}]}$ need not to be considered; the minimization of $\frac{E[\mathrm{OPT}]}{E[\mathrm{ALG}]}$ and the maximization of $\frac{E[\mathrm{ALG}]}{E[\mathrm{OPT}]}$ are both equivalent to the maximization of $E[\mathrm{ALG}]$, since $E[\mathrm{OPT}]$ is independent of the online strategy. Before discussing optimal strategies, we claim that also for $E\left[\frac{\mathrm{ALG}}{\mathrm{OPT}}\right]$ and $E[\mathrm{ALG}]$, it suffices to concentrate on strategies which exchange only when the exchange rate is the highest so far. We give the following two lemmas without proofs, which can be shown similarly to Lemma 2 . We use the same notation as in Section 4. For a given strategy $S^{*}$, let $\tilde{S}^{*}(t):=S^{*}(\bar{q}(t))$ for all $t \geq 0$. Besides, one should recall that randomization does not help for either measures as mentioned in Proposition 1. 
Lemma 5. Suppose that there exists a strategy $S^{*}$ such that $E_{F}\left[A L G_{S^{*}} / O P T\right] \geq E_{F}\left[A L G_{S} / O P T\right]$ holds for all $S$. Then, $E_{H}\left[A L G_{\tilde{S}^{*}}(t) / O P T(t)\right] \geq$ $E_{H}\left[A L G_{\tilde{S}}(t) / O P T(t)\right]$ for all $\tilde{S}$.

Lemma 6. Suppose that there exists a strategy $S^{*}$ such that $E_{F}\left[A L G_{S^{*}}\right] \geq E_{F}\left[A L G_{S}\right]$ holds for all $S$. Then, $E_{H}\left[A L G_{\tilde{S}^{*}}(t)\right] \geq E_{H}\left[A L G_{\tilde{S}}(t)\right]$ for all $\tilde{S}$.

Unlike the minimization of $E\left[\frac{\mathrm{OPT}}{\mathrm{ALG}}\right]$, we can show that for any distribution, both the maximization of $E\left[\frac{\mathrm{ALG}}{\mathrm{OPT}}\right]$ and that of $E[\mathrm{ALG}]$ lead to an optimal strategy that exchanges the whole one dollar at a certain timing. Such a strategy is referred to as RPP in [EFKT01], in which the maximization of $E[\mathrm{ALG}]$ is discussed.

We also mention the case that the given distribution belongs to a class of distributions, called IFR. According to [Ger89], we define IFR on $[m, M]$ as follows.

Definition. A distribution $F$ is said to be in IFR if $f(p) /(1-F(p))$ increases for all $p \in[m, M]$, where $f$ is the density function of $F$.

This class includes a comprehensive family of distributions such as the uniform, the normal, or a special case of Weibull distributions. For a distribution $F \in$ IFR, we prove that the optimal timing of the one-time transaction for $E\left[\frac{\mathrm{ALG}}{\mathrm{OPT}}\right]$ is earlier than that of $E$ [ALG].

Theorem 4. The strategy $S_{1}(r)=1$ for $r \in\left[r_{1}, M\right]$ and zero elsewhere maximizes $E\left[\frac{A L G}{O P T}\right]$, where $r_{1}<M$ is a maximizer of $(r-m) \int_{r}^{M} \frac{d F(p)}{p}$.

Proof. By changing the order of the integration, we have

$$
\begin{aligned}
E\left[\frac{\mathrm{ALG}}{\mathrm{OPT}}\right] & =\int_{m}^{M} \frac{m+\int_{m}^{p}(r-m) d S(r)}{p} d F(p) \\
& =m \int_{m}^{M} \frac{d F(p)}{p}+\int_{m}^{M}\left\{(r-m) \int_{r}^{M} \frac{d F(p)}{p}\right\} d S(r) \\
& \leq m \int_{m}^{M} \frac{d F(p)}{p}+\max _{m \leq r \leq M}\left\{(r-m) \int_{r}^{M} \frac{d F(p)}{p}\right\} .
\end{aligned}
$$

There exists $r_{1}<M$ which achieves the maximum and then the maximum of $E\left[\frac{\mathrm{ALG}}{\mathrm{OPT}}\right]$ is also attained for given $S_{1}$.

Theorem 5 ([EFKT01]). The strategy $S_{0}(r)=1$ for $r \in\left[r_{0}, M\right]$ and zero elsewhere maximizes $E[A L G]$, where $r_{0}$ is a maximizer of $(r-m)(1-F(r))$.

Proposition 2. If $F \in I F R$, then $r_{1}<r_{0}$.

Proof. Let $g(r):=(r-m)(1-F(r))$ and $h(r):=(r-m) \int_{r}^{M} \frac{d F(p)}{p}$. We have the derivative

$$
g^{\prime}(r)=1-F(r)-(r-m) f(r)=(1-F(r))\left\{1-(r-m) \frac{f(r)}{1-F(r)}\right\} .
$$

Since $\frac{f(r)}{1-F(r)}$ is an increasing function, the sign of $g^{\prime}(r)$ turns only once from positive to negative as $r$ grows, or remains nonnegative. Therefore $g(r)$ has a unique peak or is a non-decreasing function. We also have $h^{\prime}(r)=\int_{r}^{M} \frac{d F(p)}{p}-(r-m) \frac{f(r)}{r}$. Since $r_{1}$ is a maximizer of $h$ and 
$h^{\prime}(m)>0, h^{\prime}\left(r_{1}\right)$ is zero. Thus

$$
\begin{aligned}
g^{\prime}\left(r_{1}\right) & =1-F\left(r_{1}\right)-\left(r_{1}-m\right) f\left(r_{1}\right) \\
& =\int_{r_{1}}^{M} d F(p)-r_{1} \int_{r_{1}}^{M} \frac{d F(p)}{p} \\
& =\int_{r_{1}}^{M} \frac{p-r_{1}}{p} d F(p) \\
& >0,
\end{aligned}
$$

which implies that $g(r)$ achieves the maximum in $\left(r_{1}, M\right]$.

We give some numerical results for distributions which have already appeared as examples. As above theorems, let $r_{1}$ and $r_{0}$ denote the optimal timings for $E\left[\frac{\mathrm{ALG}}{\mathrm{OPT}}\right]$ and $E[\mathrm{ALG}]$, respectively. For the uniform distribution with $m=1$ and $M=2$, we have $r_{1}=1.46$ and $r_{0}=1.50$. As introduced in Section $1, r_{1}=2.67$ and $r_{0}=3.00$ for the case of $m=1$ and $M=5$. We have $r_{1}=1.645$ and $r_{0}=1.653$ for the Weibull distribution presented in Section 4.2. The example of a Fréchet distribution there yields $r_{1}=1.29$ and $r_{0}=1.32$. Among these examples, only the Fréchet distribution does not belong to IFR, which is a counterexample for the reverse statement of Proposition 2. $\frac{E[\mathrm{OPT}]}{E[\mathrm{ALG}]}$.

We conclude this section by providing minimax theorems with respect to $E\left[\frac{\mathrm{ALG}}{\mathrm{OPT}}\right]$ and

Theorem 6.

$$
\min _{F} \max _{A L G} E\left[\frac{A L G}{O P T}\right]=\max _{A L G} \min _{F} E\left[\frac{A L G}{O P T}\right]=\frac{1}{c_{w}} .
$$

Proof. A similar argument to the first part of the proof of Theorem 3 leads to $\max _{\mathrm{ALG}} \min _{F} E\left[\frac{\mathrm{ALG}}{\mathrm{OPT}}\right]=$ $1 / c_{w}$. The remaining is to prove

$\min _{F} \max _{\mathrm{ALG}} E\left[\frac{\mathrm{ALG}}{\mathrm{OPT}}\right]=1 / c_{w}$. By substituting WTB we have

$\min _{F} \max _{\mathrm{ALG}} E\left[\frac{\mathrm{ALG}}{\mathrm{OPT}}\right] \geq 1 / c_{w}$. Theorem 4 implies that for a given $F$, it is enough to consider strategies which exchange all at once. Let $S(r)=1$ for $r \in\left[r^{*}, M\right]$ and zero elsewhere. A minimizer is $F_{w}$ in (10). Indeed, if $r^{*}>m c_{w}$,

$$
E\left[\frac{\mathrm{ALG}}{\mathrm{OPT}}\right]=\int_{m c_{w}}^{r^{*}} \frac{m}{p} d F(p)+\int_{r^{*}}^{M} \frac{r^{*}}{p} d F(p)=\frac{r^{*}-m c_{w}}{c_{w}^{2}\left(r^{*}-m\right)}+\frac{r^{*}\left(c_{w}-1\right)}{c_{w}^{2}\left(r^{*}-m\right)}=\frac{1}{c_{w}} .
$$

Otherwise, $E\left[\frac{\mathrm{ALG}}{\mathrm{OPT}}\right]=r^{*} /\left(m c_{w}^{2}\right) \leq 1 / c_{w}$.

Lemma 7. $\min _{A L G} \max _{F} \frac{E[O P T]}{E[A L G]}=c_{w}$.

Proof. A similar argument to the first part of the proof of Theorem 3.

Lemma 8 ([EFKT01]). $\max _{F} \min _{A L G} \frac{E[O P T]}{E[A L G]}=c_{w}$.

Theorem 7.

$$
\max _{F} \min _{A L G} \frac{E[O P T]}{E[A L G]}=\min _{A L G} \max _{F} \frac{E[O P T]}{E[A L G]}=c_{w}
$$




\section{Concluding Remarks}

If one analyzes the ski-rental problem using the average-case competitive ratio instead of the conventional worst-case competitive ratio, then the optimal timing of buying skis shifts earlier [FI05]. In one-way trading the same property appears: Whereas strategy WTB waits until the exchange rate reaches the possible maximum, strategy ATB quits the transaction at a certain timing. It might be interesting to investigate whether the same property holds for other online problems in general. We should also realize a limit of average-case analysis: To carry out an average-case analysis, it is strongly required that the input sequence has a simple structure and is explicitly parameterized, such as the total time of ski trips and the maximum exchange rate.

A natural extension would be to assume other probabilistic models on the rate fluctuation, such as the geometric Brownian motion [Øks95]. It should be noticed then that one has to consider strategies with respect to time which may exchange even not at the highest rates. Please recall that Lemmas 2, 5, and 6 enable us to concentrate on strategies with respect to the highest exchange rate. Another probable difficulty would be how to define the expectation on a stochastic process.

We think that this paper is an example to illustrate that functional analysis can work in the realm of optimization of strategies/algorithms. In the most general variational problems, however, it is almost impossible to obtain an explicit solution, even though its existence is guaranteed. We suggest that for such a case, it could be helpful to narrow the solution space by executing some numerical experiments on a finite-dimensional version of the problem.

\section{Acknowledgments}

We thank Prof. Hiroyoshi Miwa for useful comments on extreme value distributions.

\section{References}

[al-99] S. al-Binali. A risk-reward framework for the competitive analysis of financial games. Algorithmica, 25(1):99-115, 1999.

[BE98] A. Borodin and R. El-Yaniv. Online Computation and Competitive Analysis. Cambridge University Press, 1998.

[Bec04] L. Becchetti. Modeling locality: A probabilistic analysis of LRU and FWF. In Proc. ESA '04, pages 98-109, 2004.

$\left[\mathrm{BLMS}^{+}\right.$06] L. Becchetti, S. Leonardi, A. Marchetti-Spaccamela, G. Schfer, and T. Vredeveld. Average-case and smoothed competitive analysis of the multilevel feedback algorithm. Math. Oper. Res., 31(1):85-108, 2006.

[CKLW01] G. Chen, M. Kao, Y. Lyuu, and H. Wong. Optimal buy-and-hold strategies for financial markets with bounded daily returns. SIAM J. Comput., 31(2):447-459, 2001.

[DLK82] M. A. H. Dempster, J. K. Lenstra, and A. H. G. Rinnooy Kan, editors. Deterministic and Stochastic Scheduling. D. Reidel Publishing Company, Dordrecht, The Netherlands, 1982. 
[EFKT92] R. El-Yaniv, A. Fiat, R. M. Karp, and G. Turpin. Competitive analysis of financial games. In Proc. FOCS 'g2, pages 327-333, 1992.

[EFKT01] R. El-Yaniv, A. Fiat, R. M. Karp, and G. Turpin. Optimal search and one-way trading online algorithms. Algorithmica, 30(1):101-139, 2001.

[EKM97] P. Embrechts, C. Klüppelberg, and T. Mikosch. Modelling Extremal Events: for Insurance and Finance. Springer-Verlag, London, UK, 1997.

[FI05] H. Fujiwara and K. Iwama. Average-case competitive analyses for ski-rental problems. Algorithmica, 42(1):95-107, 2005.

[FW98] A. Fiat and G. J. Woeginger, editors. Online Algorithms: The State of the Art. Springer-Verlag, London, UK, 1998.

[Ger89] I. B. Gertzbakh. Statistical Reliability Theory. Marcel Dekker, New York, 1989.

[GGLS08] N. Garg, A. Gupta, S. Leonardi, and P. Sankowski. Stochastic analyses for online combinatorial optimization problems. In Proc. SODA '08, pages 942-951, 2008.

[IY99] K. Iwama and K. Yonezawa. Using generalized forecasts for online currency conversion. In Proc. COCOON '99, volume 1627 of LNCS, pages 409-421. Springer, 1999.

[KP94] E. Koutsoupias and C. Papadimitriou. Beyond competitive analysis. In Proc. FOCS '94, pages 394-400, 1994.

[LPS07] J. Lorenz, K. Panagiotou, and A. Steger. Optimal algorithms for $k$-search with application in option pricing. In Proc. ESA '0\%, pages 275-286, 2007.

[Lue69] D. G. Luenberger. Optimization by vector space methods. John Wiley \& Sons Inc., New York, 1969.

[MR95] R. Motwani and P. Raghavan. Randomized Algorithms. Cambridge Univ. Press, 1995.

[MUV06] N. Megow, M. Uetz, and T. Vredeveld. Models and algorithms for stochastic online scheduling. Math. Oper. Res., 31(3):513-525, 2006.

[NR08] N. Naaman and R. Rom. Average case analysis of bounded space bin packing algorithms. Algorithmica, 50(1):72-97, 2008.

[Øks95] B. Øksendal. Stochastic differential equations: an introduction with applications. Springer-Verlag, fourth edition, 1995.

[PS06] K. Panagiotou and A. Souza. On adequate performance measures for paging. In Proc. STOC '06, pages 487-496, 2006.

[Roy88] H. L. Royden. Real Analysis. Prentice Hall, third edition, 1988.

[Sho86] P. W. Shor. The average-case analysis of some on-line algorithms for bin packing. Combinatorica, 6(2):179-200, 1986.

[SSS06] M. Scharbrodt, T. Schickinger, and A. Steger. A new average case analysis for completion time scheduling. J. ACM, 53(1):121-146, 2006.

[ST85] D. D. Sleator and R. E. Tarjan. Amortized efficiency of list update and paging rules. Commun. ACM, 28(2):202-208, 1985. 